\title{
DJ-1 interactions with $\alpha$-synuclein attenuate aggregation and cellular toxicity in models of Parkinson's disease
}

\author{
L Zondler ${ }^{1}$, L Miller-Fleming ${ }^{2,3}$, M Repici ${ }^{3}$, S Gonçalves ${ }^{2}$, S Tenreiro², R Rosado-Ramos² ${ }^{2}$ C Betzer ${ }^{4}$, KR Straatman ${ }^{5}$, PH Jensen ${ }^{4}$, \\ F Giorgini" ${ }^{* 3}$ and TF Outeiro ${ }^{*, 1,2}$
}

Parkinson's disease (PD) is a devastating neurodegenerative disorder characterized by the loss of neurons in the substantia nigra pars compacta and the presence of Lewy bodies in surviving neurons. These intracellular protein inclusions are primarily composed of misfolded $\alpha$-synuclein (aSyn), which has also been genetically linked to familial and sporadic forms of PD. DJ-1 is a small ubiquitously expressed protein implicated in several pathways associated with PD pathogenesis. Although mutations in the gene encoding DJ-1 lead to familial early-onset PD, the exact mechanisms responsible for its role in PD pathogenesis are still elusive. Previous work has found that DJ-1 - which has protein chaperone-like activity - modulates aSyn aggregation. Here, we investigated possible physical interactions between aSyn and DJ-1 and any consequent functional and pathological relevance. We found that DJ-1 interacts directly with aSyn monomers and oligomers in vitro, and that this also occurs in living cells. Notably, several PD-causing mutations in DJ-1 constrain this interaction. In addition, we found that overexpression of DJ-1 reduces aSyn dimerization, whereas mutant forms of DJ-1 impair this process. Finally, we found that human DJ-1 as well as yeast orthologs of DJ-1 reversed aSyn-dependent cellular toxicity in Saccharomyces cerevisiae. Taken together, these data suggest that direct interactions between DJ-1 and aSyn constitute the basis for a neuroprotective mechanism and that familial mutations in DJ-1 may contribute to PD by disrupting these interactions.

Cell Death and Disease (2014) 5, e1350; doi:10.1038/cddis.2014.307; published online 24 July 2014

Parkinson's disease (PD) is a fatal neurodegenerative disease characterized by the loss of neurons in the substantia nigra pars compacta. Although most cases of PD are sporadic, several rare genetic forms of the disease have been identified that have contributed greatly to our understanding of the mechanisms underlying disease pathogenesis. Misfolded $\alpha$-synuclein (aSyn) is the main protein component of Lewy bodies (LBs) - the primary pathological hallmark of $\mathrm{PD}^{1}$ - and aSyn has also been genetically linked to both familial and idiopathic forms of PD. ${ }^{2,3}$ Misfolded aSyn protein assemblies range from monomers, oligomers and protofibrils, to fibrils and, finally, to inclusion bodies, ${ }^{4}$ with precursor oligomeric forms of aSyn apparently being more toxic than larger species. ${ }^{5}$ The physiological role of aSyn remains poorly understood, but it is thought to be associated with synaptic function/plasticity, cell differentiation and vesicular trafficking. ${ }^{6}$ aSyn dysfunction has been associated with many cellular effects, including proteasome impairment, ${ }^{7}$ disruption of endoplasmic reticulumto-Golgi trafficking, ${ }^{7,8}$ increased production of reactive oxygen species (ROS) and mitochondrial dysfunction. ${ }^{9,10}$ On this evidence, several models of PD have been developed that overexpress wild-type or familial mutant versions of aSyn in organisms such as yeast, $C$. elegans, fruit flies and mice. ${ }^{2}$

Mutations in the PARK7 gene account for $\sim 1-2 \%$ of early-onset recessive $\mathrm{PD},{ }^{2,11}$ characterized by

\footnotetext{
${ }^{1}$ Department of NeuroDegeneration and Restorative Research, University Medical Center Göttingen, Göttingen, Germany; ${ }^{2}$ Instituto de Medicina Molecular, Faculdade de Medicina da Universidade de Lisboa, Lisboa, Portugal; ${ }^{3}$ Department of Genetics, University of Leicester, Leicester LE1 7RH, UK; ${ }^{4}$ Danish Research Institute of Translational Neuroscience - Dandrite, Department of Biomedicine, Aarhus University, Aarhus, Denmark and ${ }^{5}$ Centre for Core Biotechnology Services, University of Leicester, Leicester LE1 7RH, UK

${ }^{*}$ Corresponding authors: F Giorgini, Department of Genetics, University of Leicester, University Road, Leicester LE1 7RH, UK. Tel: +44 01162523485 ; Fax: +44 0116252 3378; E-mail: fg36@le.ac.uk.

or TF Outeiro, Department of NeuroDegeneration and Restorative Research, Center for Nanoscale Microscopy and Molecular Physiology of the Brain, University Medical Center Goettingen, Waldweg 33, 37073 Goettingen, Germany. Tel: +49 55139 13544; Fax: +49 55139 22693; E-mail: tiago.outeiro@ @med.uni-goettingen.de Abbreviations: $\mu \mathrm{g}$, Microgram; aSyn, $\alpha$-Synuclein; BiFC, Bimolecular fluorescence complementation; C, Celsius; C. elegans, Caenorhabditis elegans; CCD, Chargecoupled device; CFP, Cyan fluorescent protein; CNBr-Sepharose, Cyanogen bromide-activated Sepharose; $\mathrm{CO}_{2}$, Carbon dioxide; DLB, Dementia with Lewy bodies; DMEM, Dulbecco's modified Eagle's medium; DNA, Deoxyribonucleic acid; DOC, Deoxycholate; DTE, Diethyl ether; EDTA, Ethylenediaminetetraacetic acid; FCS, Fetal calf serum; GFP, Green fluorescent protein; HA, hemagglutinin; HBSS, Hank's buffered salt solution; HCl, Hydrochloric acid; HEK, Human embroynic kidney; His, Histidine; HRP, Horseradish peroxidase; HSD, Honest significant difference; HSP, Heat shock protein; LB, Lewy body; MAT, Mating type; MES, 2-(Nmorpholino)ethanesulfonic acid; min, Minute; ml, Milliliter; mM, Millimolar; Mono, monomeric; NaCl, Sodium chloride; nm, Nanometer; OD, Optical density; Oligo, Oligomeric; PBS, Phosphate-buffered saline; PD, Parkinson's disease; PFA, Parafomaldehyde; PGK, Phosphoglycerate kinase; PVDF, Polyvinylidene fluoride; RFP, Red fluorescent protein; RIPA, Radio-immunoprecipitation assay; RNA, Ribonucleic acid; ROS, Reactive oxygen species; r.p.m., Rounds per minute; RT, Room temperature; S. cerevisiae, Saccharomyces cerevisiae; SDS, Sodium dodecyl sulfate; SDS-PAGE, Sodium dodecyl sulfate polyacrylamide gel electrophoresis; S.E.M., Standard error of the mean; SOD1, Superoxide dismutase 1; TBST, Tris buffered saline with Tween; W, Watt; WT, Wild-type

Received 13.3.14; revised 11.6.14; accepted 13.6.14; Edited by D Bano
} 
levodopa-responsive parkinsonism. PARK7 encodes for DJ-1, a small conserved ubiquitously expressed protein of 189 residues primarily localized in the cytoplasm, but also found in the nucleus and associated with mitochondria. ${ }^{12-14}$ Crystallography has revealed that DJ-1 is a homodimer, which appears to be critical for its normal physiological function. ${ }^{15-17}$ A diverse set of potential functions have been suggested for DJ-1, including roles as an oxidative stress sensor (via a cysteine residue at position 106, C106), a protein chaperone, a protease, an RNA-binding protein, a transcription regulator, a regulator of mitochondria function and a regulator of autophagy. ${ }^{18}$ It is unclear which, if any, of these processes underlie DJ-1-dependent pathogenesis in PD.

Previous studies reported that DJ-1 can modulate the aggregation and toxicity of aSyn. ${ }^{19-21}$ Here, we investigated this relationship further and found that direct interactions between DJ-1 and monomeric/oligomeric aSyn are likely to underlie this process. Furthermore, we observed that familial PD mutations disrupt the interaction between DJ-1 and aSyn and abrogate the protective effects of DJ-1. In total, our work supports enhanced DJ-1 activity as an important target for therapeutic intervention in PD.

\section{Results}

DJ-1 binds monomeric and oligomeric aSyn in vitro. Although previous studies found that DJ-1 can modulate aSyn aggregation and cytotoxicity, ${ }^{22}$ the nature of the possible physical interaction underlying this process is unknown. Thus, we sought to shed light into the interplay between the two proteins. To investigate whether an interaction between endogenous DJ-1 and aSyn is detected in the mouse brain, we generated C57BL/6 wild-type (WT) mouse brain homogenate and performed co-immunoprecipitation with anti-aSyn antibodies (Figure 1a). An interaction between DJ-1 and aSyn was detected indicating that DJ-1 and aSyn can specifically interact with each other at endogenous concentrations in the brain (Figure 1a).

Next, to determine if the interaction between DJ-1 and aSyn depends upon the aSyn aggregation species, we isolated purified aSyn monomers and oligomers as previously described, ${ }^{23}$ and incubated these preparations with extracts from HEK293 cells expressing myc-tagged DJ-1. Co-immunoprecipitation with anti-aSyn antibodies showed that both monomeric and oligomeric forms of aSyn interacted with DJ-1 with equivalent efficiencies (Figure 1b). These data indicate that DJ-1 physically interacts with soluble monomeric and oligomeric forms of aSyn in vitro.

DJ-1 interacts with aSyn in living cells. As DJ-1 can bind monomeric and oligomeric forms of aSyn in vitro, we next explored the interaction between the two proteins in living cells using bimolecular fluorescence complementation (BiFC), a powerful approach used extensively by our groups. 5,17 BiFC takes advantage of the reconstitution of non-fluorescent fragments of fluorescent proteins (GFP, RFP, Venus, etc.) to study protein-protein interactions in living cells. ${ }^{24}$ In this study, BiFC constructs encoding either DJ-1 or aSyn tagged with halves of GFP or Venus were co-transfected into HEK293 cells (Figure 2). aSyn dimerization

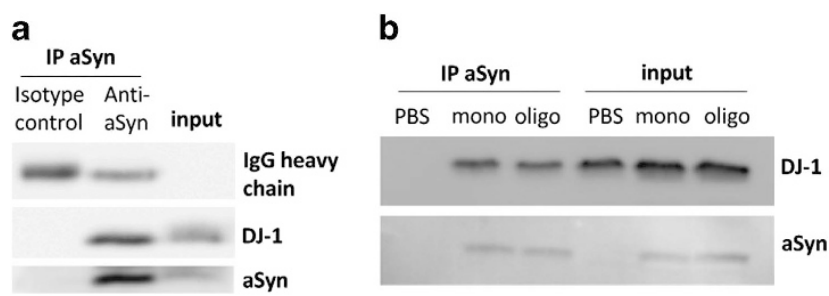

Figure 1 DJ-1 binds monomeric and oligomeric aSyn in vitro. (a) Coimmunoprecipitation of aSyn and DJ-1 in mouse brain homogenate demonstrates that DJ-1 and aSyn interact at endogenous levels. An isotype IgG control was performed to confirm the specificity of the interaction. One representative blot of three replicates is shown. (b) Co-immunoprecipitation of monomeric (mono) and oligomeric (oligo) aSyn with DJ-1 from lysates of DJ-1 overexpressing cells demonstrates that DJ-1 can bind both monomeric and oligomeric aSyn species in vitro. PBS instead of aSyn mixed with the lysate was used as negative control. The input lane is $2 \%$ of the sample prior to immunoprecipitation. One representative blot of three replicates is shown

can be preferentially detected using constructs in an antiparallel orientation in the BiFC assay ${ }^{5}$ with the N-terminal portion of GFP located at the N-terminus of aSyn and the C-terminal portion of GFP at the C-terminus of aSyn. Here, we utilized fusions of aSyn with fragments of Venus, because of the improved fluorescence emission of this protein compared with GFP. ${ }^{25}$ DJ-1 is known to form homodimers ${ }^{16,26}$ and we previously characterized this dimerization in living cells using the BiFC assay. ${ }^{17}$ For the current study, we took advantage of the previously employed DJ-1 BiFC constructs, which encode DJ-1 tagged at its C-terminus with GFP or CFP halves (DJ-1-CFPC and DJ-1-GFPN; Figure 2). Fluorophore reconstitution has been shown to also occur for the combinations of Venus and GFP or CFP, respectively. ${ }^{25}$

To quantify the BiFC efficiency between DJ-1 and aSyn, we normalized the Venus/GFP emissions by the emissions of RFP or mCherry produced from a co-transfected expression plasmid. After subtraction of the background fluorescence, the intensity ratios between GFP and RFP fluorescence were calculated for every cell positive for red fluorescence, as previously described. ${ }^{17}$ Homodimeric interaction of DJ-1 or aSyn was confirmed by co-transfection of the two DJ-1 WT constructs and the two aSyn constructs, respectively (Figures $2 \mathrm{~b}$ and $\mathrm{c}$ ). Transfection of only one of the two BiFC constructs or the empty vector (pcDNA3.1+) did not lead to emission of green fluorescence (Figure 2c), whereas co-transfection of aSyn (VN-Syn) and DJ-1 (DJ-1-CFPC) BiFC constructs resulted in reconstitution of the fluorophores, indicating a direct interaction between these two proteins in living cells (Figures $2 b$ and c). For the combination of Syn-VC and DJ-1GFPN, no fluorophore reconstitution was observed (Figures $2 \mathrm{~b}$ and $\mathrm{c}$ ), indicating that the interaction between $\mathrm{DJ}-1$ and aSyn can only be detected when the tags are present in a specific orientation, as previously observed for other proteins in BiFC experiments. ${ }^{5}$ Importantly, this result also confirms the specificity of the interaction, because the two fragments of the fluorescent proteins are present in the same cell but do not reconstitute the fluorophore if the orientation is not adequate. Expression of the DJ-1 and aSyn BiFC constructs was confirmed by immunoblotting for each experiment (Figure 2d). The reduced expression of Syn-VC construct compared to that of VN-Syn construct has been previously 
reported, ${ }^{5}$ and is likely because of the effects of the location of the tag on the protein. However, as shown for the VN-Syn/ Syn-VC combination, this does not constrain the BiFC signal. Thus, our results indicate that DJ-1 and aSyn can indeed interact - likely directly, given the nature of the BiFC assay - in living cells.

Familial PD mutations abrogate interactions between DJ-1 and aSyn. To investigate whether the interaction between DJ-1 and aSyn was influenced by PD-causing mutations in one of the partners, HEK293 cells were cotransfected with combinations of WT VN-Syn and familial mutant DJ-1-CFPC constructs or familial mutant constructs of VN-Syn with WT DJ-1-CFPC, respectively. Quantification of the BiFC efficiency was performed as described above for each BiFC combination. The DJ-1 mutants L166P, M26I, $\mathrm{L} 10 \mathrm{P}$ and P158 $\Delta$ led to a dramatic reduction in the BiFC signal, thus indicating that the interaction with aSyn is abrogated compared to WT DJ-1 $(P<0.0001$; Figure 3a). The C106A, E64D and K130R mutations led to a slight but significant change in DJ-1/aSyn interactions (Figure 3a). Interestingly, the familial aSyn mutations A30P, E46K and A53T did not significantly alter the BiFC signal as compared to WT aSyn (Figure 3b).

Immunoblotting experiments showed that DJ-1 and aSyn BiFC constructs were highly expressed as WT proteins in HEK293-transfected cells, as were the mutant aSyn proteins (A30P, E46K, A53T) and the E64D, K130R and C106A DJ-1 mutants (Figure 3c). The L166P, M26I, L10P and P158 DJ-1 mutants were expressed at lower levels, which is consistent with previous findings showing that certain forms of mutant $\mathrm{DJ}-1$ are less stable and more rapidly degraded than the WT protein. ${ }^{17}$ These data indicate that for this subset of DJ-1 familial mutations, the decrease in interactions with aSyn is due - at least in part - to decreased expression levels, which is likely representative of what occurs in patients. Nonetheless, these results indicate that familial mutations in DJ-1 can dramatically abrogate interactions with aSyn in living cells.

DJ-1 modulates aSyn oligomerization. To investigate the impact of DJ-1 on aSyn oligomerization, WT and mutant (L10P, M26I, P1584, L166P) DJ-1-myc plasmids were transiently transfected, along with the mCherry transfection control plasmid, into $\mathrm{H} 4$ cells stably expressing VN-Syn and Syn-VC. This BiFC model of aSyn dimerization/oligomerization was previously described by our group and results in a clear fluorescence signal distributed throughout the cell ${ }^{5}$ (Figure 4a). When H4 VN-Syn/Syn-VC cells were transfected with myc-tagged WT DJ-1, a significant $\sim 22 \%$ decrease in the BiFC signal was observed in comparison to cells transfected with the empty vector control (pcDNA3.1+) (Figures $4 \mathrm{~b}$ and $\mathrm{c} ; P<0.01$ ), indicating a decrease in aSyn oligomerization because of WT DJ-1. L10P, M26I and P158 mutant forms of DJ-1 failed to significantly impair aSyn oligomerization (Figures $4 \mathrm{~b}$ and $\mathrm{c}$ ), suggesting that these DJ-1 mutations lead to a loss of DJ-1 chaperone function. L166P mutant DJ-1, however, reduced the aSyn BiFC signal to a similar extent as WT DJ-1 (Figures $4 \mathrm{~b}$ and $\mathrm{c}$; $P<0.05$ ).
Expression of the transfected DJ-1 constructs was confirmed by immunoblotting (Figure 4d), supporting the finding that the familial mutations impair the capability of DJ-1 to antagonize aSyn dimerization/oligomerization. Interestingly, the M26I, P1584 and L166P mutants were expressed at levels similar to WT DJ-1, which is in contrast to our immunoblotting data above as well as past studies that found decreased stability of these proteins. ${ }^{17}$ This is likely because of the $\mathrm{N}$-terminal myc tag stabilizing these proteins, thereby permitting the direct testing of the effect these mutations have on aSyn oligomerization, independent of protein levels. In the case of L10P DJ-1, expression was lower than the WT DJ-1, which is consistent with previous studies. ${ }^{17}$

DJ-1 reduces aSyn aggregation and cellular toxicity in yeast. Since the initial report on aSyn aggregation and toxicity in budding yeast, ${ }^{9}$ this organism has been extensively used to study the mechanisms underlying PD and other neurodegenerative disorders. ${ }^{27,28} S$. cerevisiae has four genes that belong to the DJ-1 superfamily (HSP31, HSP32, HSP33, HSP34). ${ }^{29}$ We found that co-expression of each of these four yeast genes reduced aSyn toxicity in yeast as indicated by spotting assays (Figure 5a). To investigate whether human DJ-1 has the same effect on aSyn toxicity, we repeated the experiment co-expressing human DJ-1 and aSyn in yeast. Concordantly, we found that aSyn toxicity is reduced by human DJ-1 (Figure $5 \mathrm{~b}$ ). Immunoblotting showed that the protein levels of aSyn did not change in the presence of the Hsps, demonstrating that the effect on toxicity was not simply because of decreased levels of aSyn in the cell (Figure 5c). Next, we performed a sucrose velocity gradient assay to separate proteins according to their molecular weight, as we previously described. ${ }^{30}$ Overexpression of all the DJ-1 superfamily members reduced the high molecular weight aSyn species (Figure $5 \mathrm{~d}$ ). This was further corroborated by counting the number of cells with aSyn inclusions by fluorescence microscopy (Figures $5 e$ and $\mathrm{f}$ ). We observed a $\sim 15 \%$ reduction in the number of cells with inclusions when the Hsps were co-expressed $(P<0.01)$, which is in agreement with our data obtained in mammalian cells showing that DJ-1 modulates aSyn oligomerization.

\section{Discussion}

aSyn plays an important role in the pathogenesis of several neurodegenerative diseases including PD and dementia with Lewy bodies. ${ }^{31-34}$ Indeed, aggregated aSyn is the major component of Lewy bodies, protein inclusions that are found in the brains of PD and DLB patients. ${ }^{31}$ Moreover, mutations in or increased dosage of the aSyn-encoding gene lead to autosomal dominant familial PD. However, whether aSyn aggregation per se is cytotoxic and causes neurodegeneration, or the aggregation is an attempt to shield the neurons from toxic aSyn molecules of lower order, is still controversial. ${ }^{3,5,35-38}$ $\mathrm{DJ}-1$ is a ubiquitously expressed protein known to regulate the cellular response to oxidative stress by various mechanisms, including regulation of gene expression and mitochondrial integrity, induction of survival pathways and protein refolding. ${ }^{12,19,22,39-42}$ Mutations in the gene encoding DJ-1 can cause autosomal recessive PD, ${ }^{1,43}$ although the underlying 
a

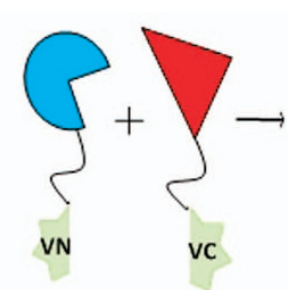

b
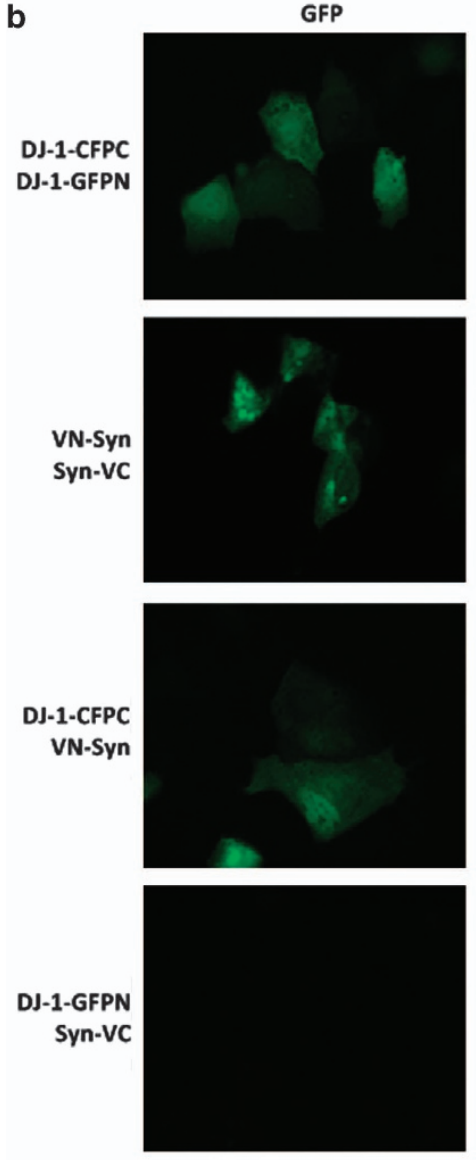

c

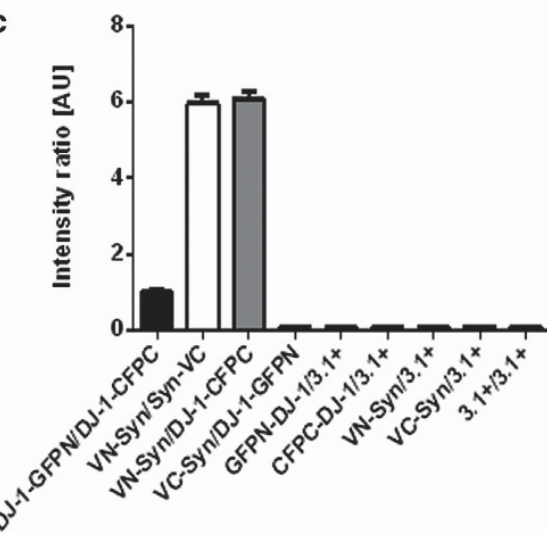

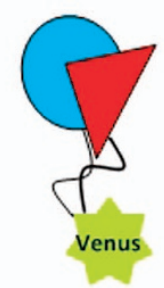

Hoechst 33258
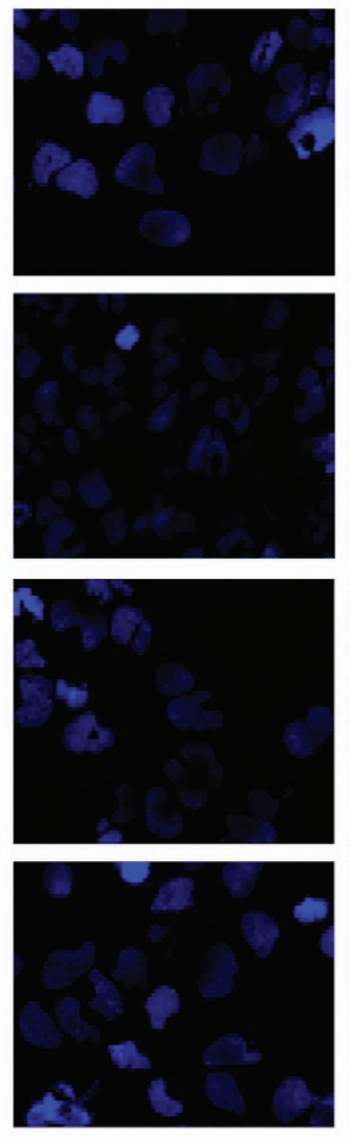
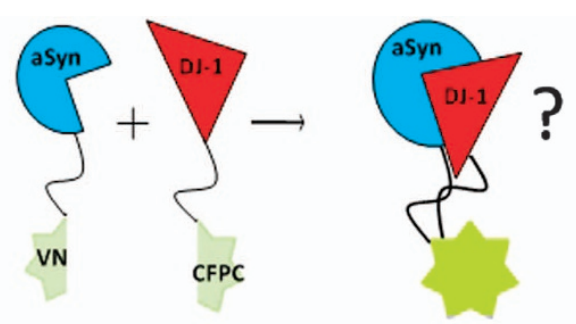

merge
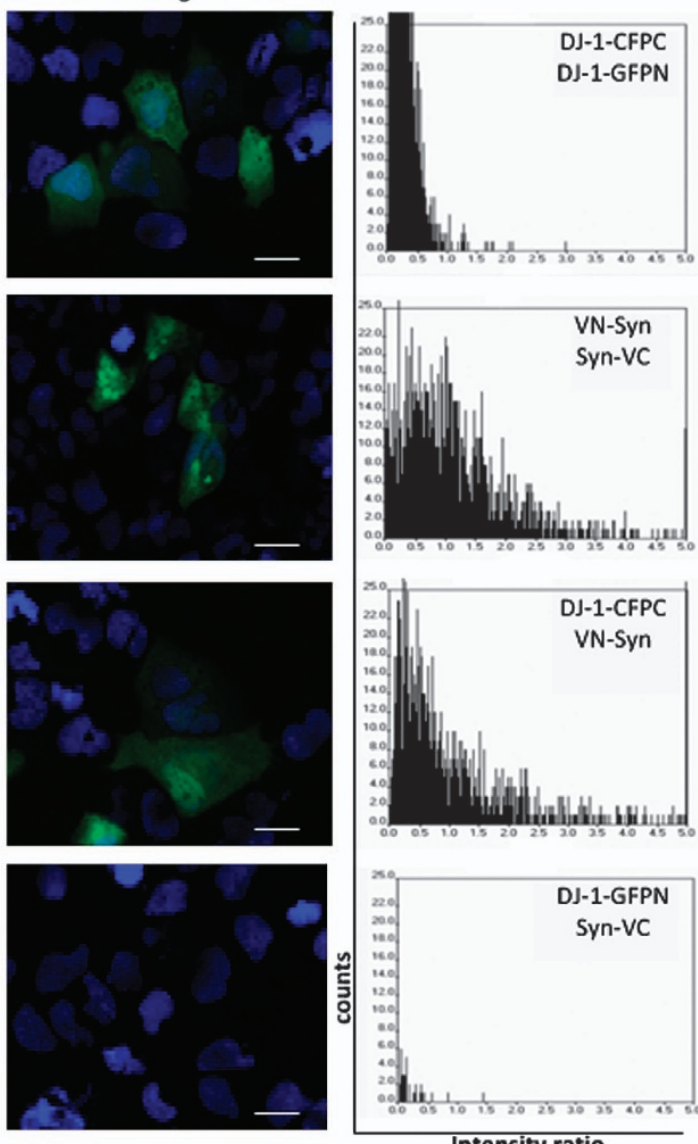

d

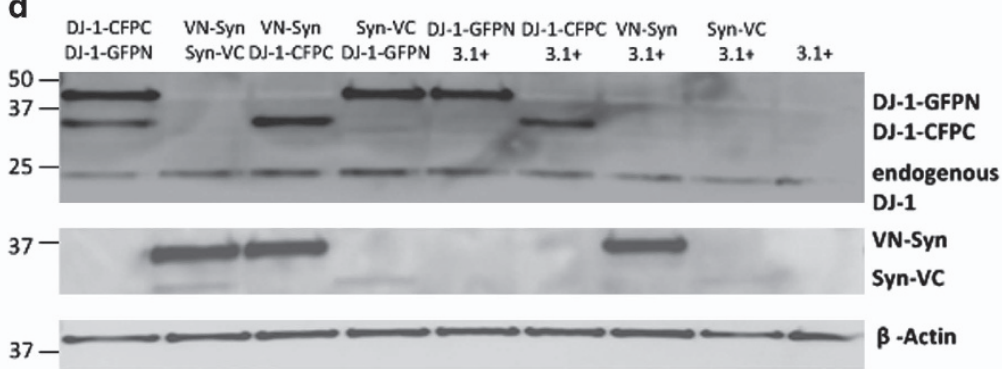


pathology leading to the development of PD is complex and various mechanisms are thought to be involved. ${ }^{31,44,45}$ Indeed, mitochondrial impairment and oxidative stress as well as protein misfolding are key events, but it is still not clear whether they are a cause or a consequence of the disease process.

DJ-1 has been found to be involved in these pathways in a neuroprotective manner. In line with our findings, DJ-1 has been shown to prevent protofibril formation by monomeric aSyn in vitro ${ }^{19}$ and reduce aSyn aggregation and toxicity. ${ }^{22}$ In addition, we also recently found that $\mathrm{DJ}-1$ modulates mutant huntingtin aggregation in vitro and in vivo, in different models of Huntington's disease. ${ }^{46}$ In all of the reported studies, the chaperone-like activity of DJ-1 was dependent on the oxidative environment ${ }^{46}$ and, in particular, on the oxidation state of $\mathrm{C} 106 .{ }^{47}$ Complementing these observations, our experiments find that the interaction of C106A mutant DJ-1 and aSyn is significantly impaired, further supporting the importance of the oxidation state of this residue for DJ-1 function and activation. Furthermore, DJ-1 has been found to bind copper ions at $\mathrm{C} 106$, which under critical conditions can be transferred to SOD1 (superoxide dismutase 1), thereby activating its catalytic function. ${ }^{48}$ This is thought to take place via a direct copper chaperone-like interaction between DJ-1 and SOD1. ${ }^{49}$

On the basis of the finding that DJ-1 prevents aSyn fibril formation, ${ }^{19}$ many reasoned that a direct interaction between the two proteins was likely to take place. However, previous attempts have failed to show a direct interaction between DJ-1 and aSyn, $^{50}$ possibly because of the need of additional cofactors to stabilize the interaction. Therefore, our study demonstrates for the first time that DJ-1 and aSyn can directly interact in vitro and in living cells. As both the BiFC assay and our in vitro approaches demonstrate an interaction of DJ-1 with monomeric aSyn, our work is consistent with the idea that the DJ-1-aSyn interaction is an early event in the aSyn aggregation process. ${ }^{19}$
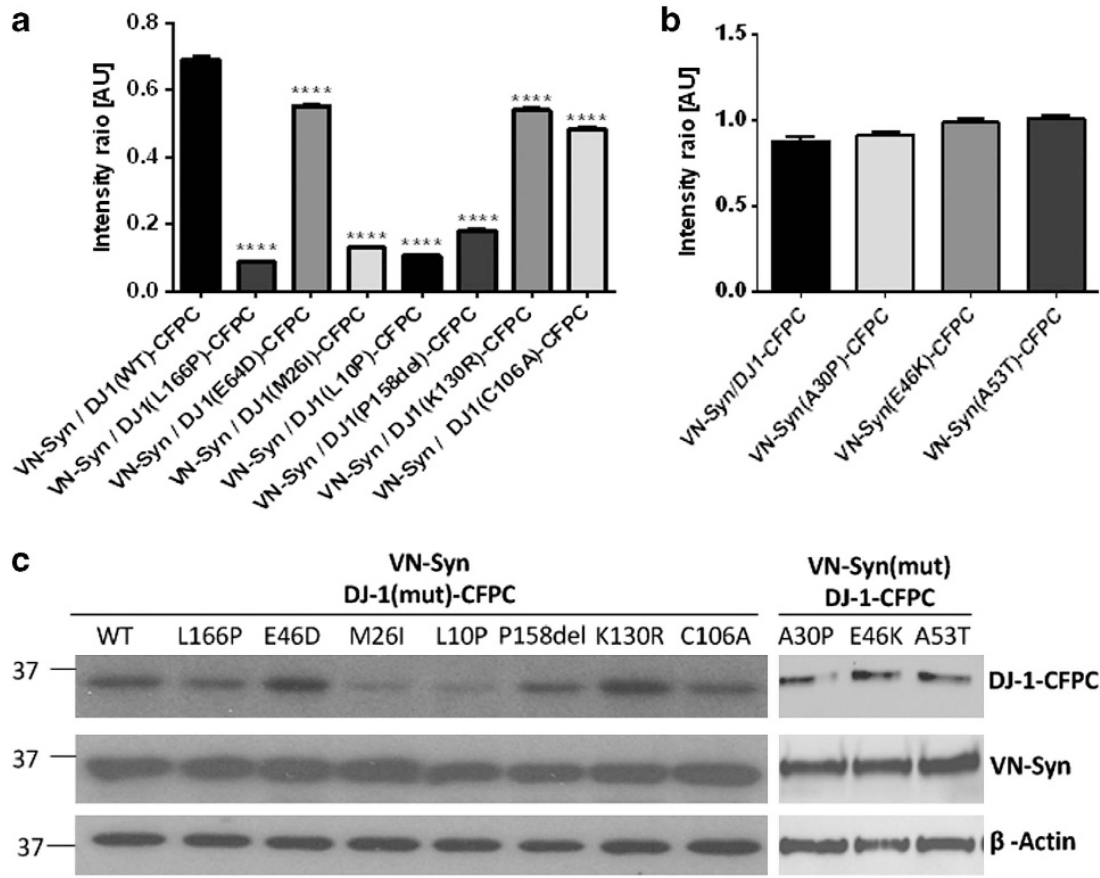

Figure 3 Familial PD DJ-1 mutations decrease DJ-1/aSyn interactions in living cells. (a) Effect of L166P, E64D, M26I, L10P, P158 A, K130R and C106A DJ-1 mutations on the efficiency of fluorescence complementation between DJ-1 and aSyn BiFC constructs. The histogram shows the average ratio intensity (Green/Red) + S.E.M. per well. All the investigated DJ-1 mutations constrain the interaction efficiency of DJ-1 with aSyn significantly as indicated by decreased fluorescence intensity ratios compared to the DJ-1 (WT)/aSyn (WT) combination. ${ }^{* * *} P<0.0001$. (b) Effect of A30P, E46K and A53T aSyn mutations on the efficiency of fluorescence complementation between DJ-1 and aSyn BiFC constructs. The histogram shows the average ratio intensity + S.E.M. per well. The ratio intensity of the BiFC signal from HEK293 cells co-transfected with VN-Syn (WT and mutants) and DJ-1(WT)-CFPC are not significantly different from one another, indicating that familial aSyn mutations (A30P, E46K, A53T) do not impair the interaction of aSyn with DJ-1. (c) Immunoblots of the lysates obtained from the transfected cell populations used in A. Upper panel: anti DJ-1 antibody, Middle panel: anti aSyn antibody, Lower panel: anti Tubulin (left panel); anti $\beta$-actin antibody (right panel)

Figure 2 DJ-1 interacts with aSyn in living cells. (a) Schematic representation of the BiFC assay. The interaction of two proteins of interest (DJ-1 and aSyn) tagged with non-fluorescent fragments of fluorescent proteins (Venus or CFP) drives the reconstitution of the functional fluorophore resulting in fluorescence emission. (b) Representative sections showing the fluorescence complementation signal of DJ-1-GFPN/DJ-1-CFPC, VN-Syn/Syn-VC, VN-Syn/DJ-1-CFPC and Syn-VC/DJ-1-GFPN in living cells. Scale bar: $100 \mu \mathrm{m}$. The distribution of ratios between GFP and mCherry emissions in individual cells co-transfected with plasmids encoding the proteins is shown in each graph. (c) The histogram shows the average ratio intensity for the indicated plasmid combinations (+ S.E.M.). The combinations of the BiFC constructs DJ-1-GFPN/DJ-1-CFPC, VN-Syn/Syn-VC and VN-Syn/DJ-1-CFPC result in a fluorescence complementation signal indicated by positive intensity ratios when normalized to the mCherry transfection control. The same constructs combined with an empty vector control $(3.1+)$ or transfection of the empty vector control alone do not result in positive fluorescence intensity ratios, indicating that fluorescence is only caused by the interaction of the two complementary constructs. (d) Immunoblotting analysis of the protein lysates obtained from transfected cell populations used in the BiFC experiment show the expression of the tested BiFC constructs, Upper panel: anti DJ-1 antibody, Middle panel: anti aSyn antibody, Lower panel: anti $\beta$-actin antibody 


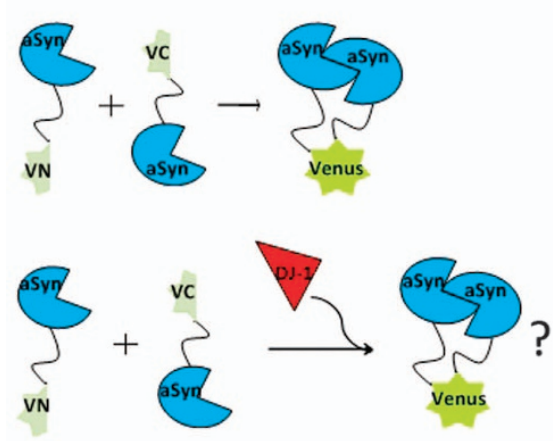

C

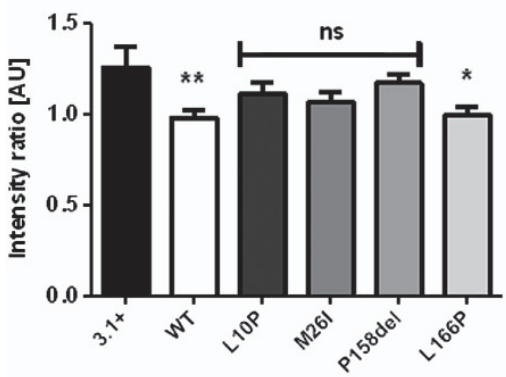

b
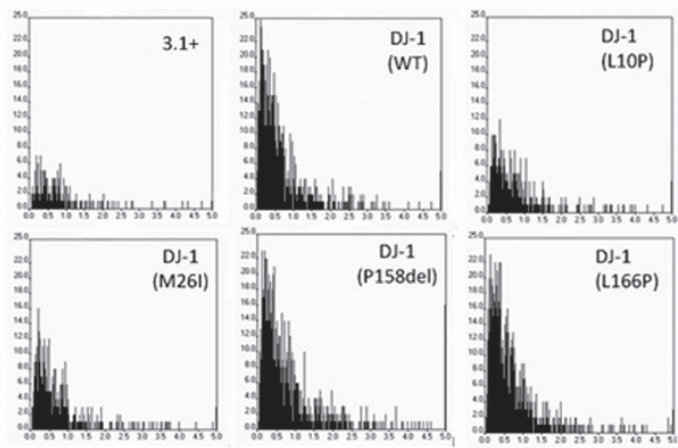

d
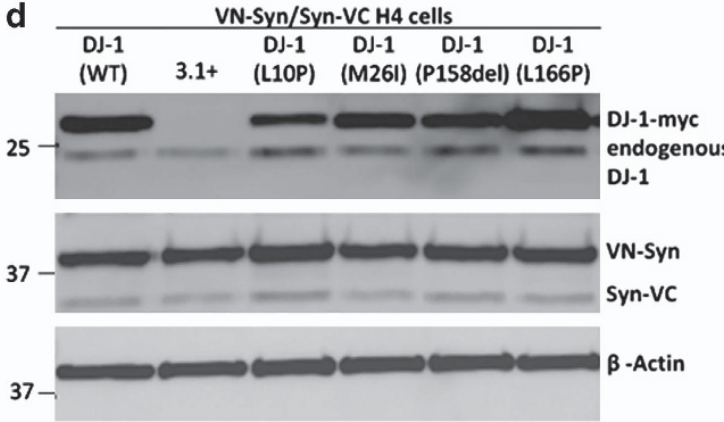

Figure 4 DJ-1 modulates aSyn oligomerization. (a) Schematic representation of the BiFC model of aSyn oligomerization used in this experiment. H4 cells stably expressing VN-Syn/Syn-VC were used to examine the impact of transiently transfected DJ-1 on aSyn oligomerization. (b) The impact of DJ-1 (WT and mutants, as indicated in the graphs) on aSyn oligomerization was examined. The distribution of intensity ratios between Venus and mCherry emissions in individual cells transiently transfected with the plasmid encoding myc-tagged DJ-1 (WT or mutant) or an empty vector control (3.1+) together with the mCherry control plasmid is shown in each graph. (c) The histogram shows the average ratio intensity + S.E.M. per well. A decrease was observed when VN-aSyn/aSyn-VC expressing H4 cells were transfected with DJ-1 (WT) compared to the empty vector control $3.1+(P<0.01)$ indicating that WT DJ-1 interferes with aSyn oligomerization. DJ-1 mutants (L1OP, M26I, P158 $)$ failed to impair aSyn oligomerization to the same degree, while the L166P mutant significantly decreased the BiFC signal $(P<0.05)$. (d) Immunoblot confirming the expression of transfected myc-DJ-1 constructs, as well as the stable expression of VN-Syn and Syn-VC. Upper panel: anti DJ-1 antibody, Middle panel: anti aSyn antibody, Lower panel: anti $\beta$-actin antibody

As mutations in the genes encoding these two proteins are implicated in familial PD, ${ }^{1}$ we hypothesized that these familial mutations could affect the interaction between the two proteins. Indeed, we demonstrate that familial PD mutations of DJ-1 affect this interaction. Furthermore, we show that DJ-1 reduces aSyn dimerization whereas familial $P D$ mutant versions of $\mathrm{DJ}-1$ fail to antagonize aSyn dimerization to the same degree. The mechanisms by which DJ-1 mutations cause the disease are not known, and an impairment of the interaction between DJ-1 and aSyn could provide a mechanistic explanation. Interestingly, the familial aSyn mutations interrogated in our study (A30P, E46K, A53T) did not abrogate interactions with WT DJ-1 by BiFC. To date, protein-protein interactions of aSyn have mostly been investigated in terms of aggregation (e.g. aggregation with tau or $A \beta)^{51,52}$ or membrane association, ${ }^{53}$ but not regarding potentially neuroprotective players in the PD context.

In contrast to the familial aSyn mutations, familial DJ-1 mutations - particularly L166P, M26I, L10P and P158 - were found to abrogate the interaction between DJ-1 and aSyn. These mutations impair DJ-1 homodimerization, ${ }^{17,54,55}$ suggesting that the functionality of the protein is perturbed. Moreover, these mutants increase the degradation and turnover rate of DJ $1,{ }^{17,54,55}$ which explains the lower protein levels detected in these experiments. Our data suggest that the recessive familial mutations that impair dimerization and protein stability of DJ-1 also hinder the interaction between DJ-1 and aSyn, which is in agreement with several familial PD mutations affecting normal DJ-1 function. Notably, E64D DJ-1 is stable and dimerizes in a manner similar to $\mathrm{WT}^{17}$ and it is thus still unclear how this mutation might cause PD; our results show, however, that the E64D mutation restrains the interaction of DJ-1 with aSyn. The C106A mutation abolishes DJ-1 oxidation at this residue, which has been shown to be crucial for its chaperone activity. ${ }^{47}$ Therefore, our finding that the interaction of C106A DJ-1 with aSyn is significantly decreased compared to WT DJ-1 supports the hypothesis that DJ-1 acts as a chaperone on misfolded aSyn. As the K130R mutation - which prevents DJ-1 SUMOylation - also impairs the interaction with aSyn, the chaperone-like activity of DJ-1 may not only be dependent on oxidation but also upon SUMOylation.

As aSyn misfolds and aggregates in PD, and DJ-1 displays chaperone-like functions, ${ }^{19,46,49}$ we hypothesized that DJ-1 may modulate aSyn aggregation. Thus, we investigated the effect of DJ-1 on aSyn oligomerization using BiFC and observed that WT DJ-1 significantly reduces aSyn oligomerization in living cells. Our findings support previous studies that found DJ-1 prevented aSyn protofibril formation from monomeric aSyn ${ }^{19}$ and reduced aSyn aggregation. ${ }^{47}$ Taken together, these results suggest that DJ-1 may prevent aSyn aggregation by interfering with the initial steps in the aggregation process of di- or oligomerization. L10P, M26I 
a

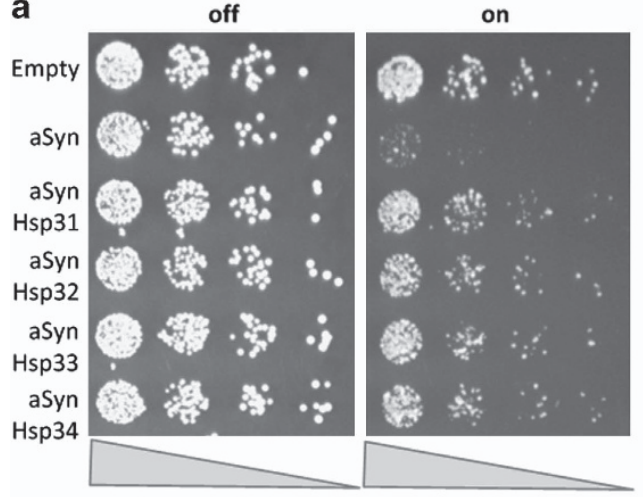

b

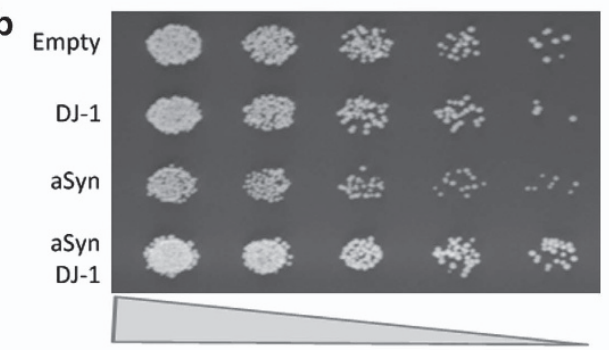

e
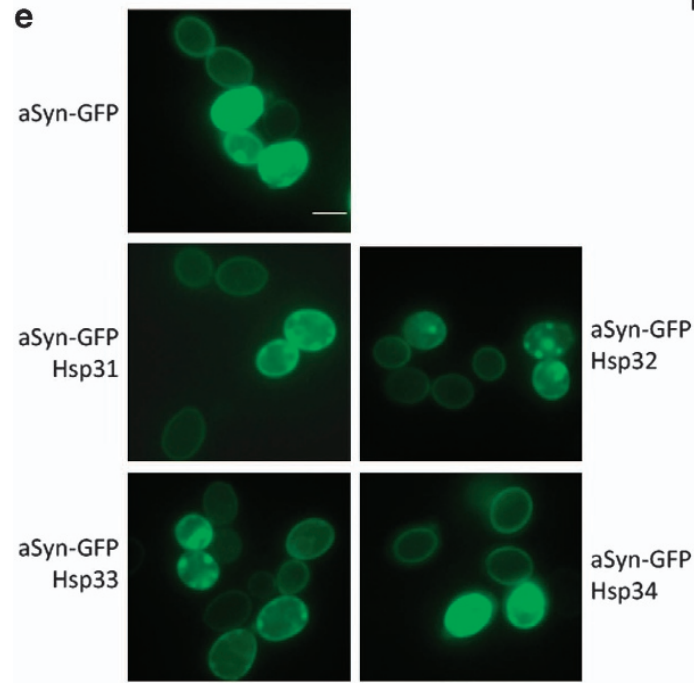

C

Empty aSyn aSyn aSyn aSyn aSyn Hsp31 Hsp32 Hsp33 Hsp34

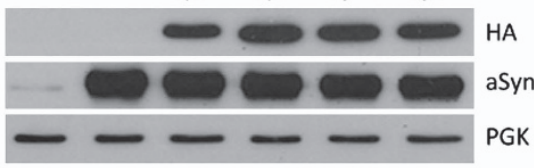

d

fraction $1 \begin{array}{lllllllll}1 & 2 & 3 & 4 & 5 & 6 & 7 & 8 & 9\end{array}$

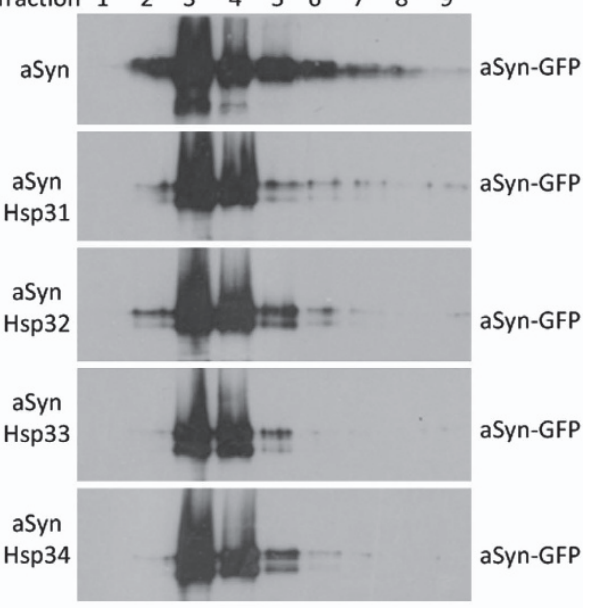

f

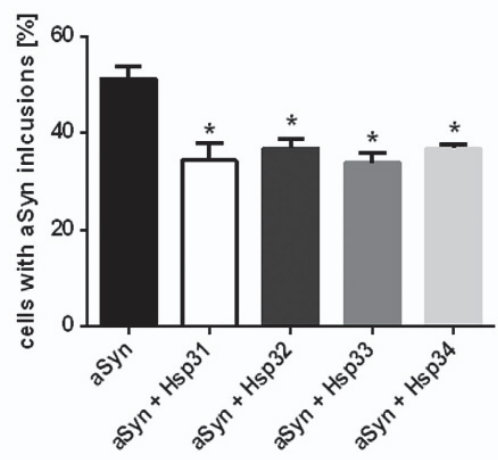

Figure 5 DJ-1 suppresses aSyn toxicity and aggregation in yeast. (a) Spotting assay of yeast cultures co-expressing aSyn and Hsp31, Hsp32, Hsp33 or Hsp34. Cultures were adjusted to $\mathrm{OD}_{600 \mathrm{~nm}}=0.1$, fivefold serially diluted and spotted onto media plates containing either glucose (aSyn and Hsps expression is repressed, 'OFF') or galactose (expression is induced, 'ON'). The spotting assay demonstrates that aSyn expression significantly reduces yeast growth and that co-expression of Hsp31, Hsp32, Hsp33 and Hsp34 reverses this toxicity. (b) Spotting assay of yeast cultures expressing aSyn and/or DJ-1. Cultures were adjusted to $\mathrm{OD}_{600 \mathrm{~nm}}=0.1$, fivefold serially diluted and spotted onto media plates to observe aSyn toxicity. This spotting assay confirms that aSyn expression significantly reduces yeast growth and that co-expression of human DJ-1 reverses the toxicity. (c) Immunoblotting demonstrates the expression levels of aSyn-GFP, Hsp31, Hsp32, Hsp33, and Hsp34 after $7 \mathrm{~h}$ of induction of aSyn expression. PGK was used as loading control. (d) Co-expression of aSyn with Hsp31, Hsp32, Hsp33 or Hsp34 reduces the high molecular weight aSyn species formed in yeast as analyzed by a sucrose velocity gradient and subsequent immunoblotting $7 \mathrm{~h}$ after induction of aSyn expression. (e) Representative images showing the intracellular localization of aSyn-GFP expressed alone or with Hsp31, Hsp32, Hsp33 or Hsp34. Scale bar: $5 \mu \mathrm{m}$. (f) The histogram shows the mean percentage of yeast cells containing aSyn inclusions + S.E.M., assessed by fluorescence microscopy $7 \mathrm{~h}$ after induction of aSyn expression. Co-expression of Hsp31, Hsp32, Hsp33 or Hsp34 significantly decreases the number of aSyn inclusions $(P<0.05)$

and P158 $\Delta$ DJ-1 are unable to decrease aSyn dimerization, clearly suggesting a loss of the chaperone DJ-1 function in these mutants. Unexpectedly, the L166P DJ-1 mutant significantly reduced aSyn oligomerization. Notably, the L166P protein is highly expressed in these experiments, likely as a result of protein stabilization because of the $\mathrm{N}$-terminal myc tag. This suggests that this stabilized form of L166P is functional, and thereby is able to modulate aSyn aggregation.
Our findings in yeast, with human DJ-1 as well as the four yeast DJ-1 orthologs, further suggest that the chaperone function of $D J-1$ is beneficial, as aSyn toxicity was ameliorated alongside with a reduction in aggregation.

In summary, we provide the first evidence that a direct interaction between aSyn and DJ-1 could be an important neuroprotective mechanism in the context of $P D$, and that the disruption of these interactions can contribute to the pathology 
caused by DJ-1 mutations. These observations support the notion that pharmacological interventions aimed at enhancing DJ-1 activity may provide a relevant therapeutic option in PD and in other synucleinopathies.

\begin{abstract}
Materials and Methods
Immunoprecipitation of aSyn monomers and oligomers. HEK293 cells were transiently transfected by Neon (Invitrogen, Carlsbad, CA, USA) with myc-DJ-1 using $7.5 \mu \mathrm{g}$ DNA and $1100 \mathrm{mV}$ for $20 \mathrm{~ms}, 2$ pulses. After $24 \mathrm{~h}$, the cells were trypsinized, collected by centrifugation, washed in Hank's buffered salt solution (HBSS) and lysed in RIPA buffer (50 mM Tris, $150 \mathrm{mM} \mathrm{NaCl}, 1 \%$ Triton $\mathrm{X}-100,2 \mathrm{mM}$ EDTA, $0.5 \%$ DOC, $0.1 \%$ SDS, Complete protease inhibitor cocktail mix, Roche, Basel, Switzerland). aSyn oligomers were produced by incubation of $10 \mathrm{mg} / \mathrm{ml}$ recombinant aSyn in phosphate-buffered saline (PBS) for $30 \mathrm{~min}$ on ice and isolated by gel filtration as previously described. ${ }^{23} 2 \mu \mathrm{g}$ of aSyn oligomers or monomers were mixed with $1.5 \mathrm{mg} / \mathrm{ml} \mathrm{HEK} \mathrm{myc-DJ-1} \mathrm{lysate} \mathrm{in}$ PBS $+0.5 \%$ TX-100 and allowed to interact over night at $4{ }^{\circ} \mathrm{C}$ with rotation. $2 \%$ of input sample was isolated prior to addition of $30 \mu \mathrm{l} \mathrm{CNBr-sepharose} \mathrm{coupled} \mathrm{to}$ anti-aSyn (ASY-1), an affinity purified rabbit antibody against human aSyn as previously described. ${ }^{56}$ Samples were incubated at $4^{\circ} \mathrm{C}$ with rotation for $2 \mathrm{~h}$. Sepharose beads were isolated by centrifugation at 1500 r.p.m. for $1 \mathrm{~min}$ at $4{ }^{\circ} \mathrm{C}$ and washed five times in PBS $+0.5 \%$ TX-100. Elution was obtained by adding non-reducing SDS-PAGE loading buffer $(50 \mathrm{mM}$ tris pH 6.8, 4\% SDS, $40 \%$ glycerol, bromphenol blue) and incubated at $37^{\circ} \mathrm{C}$ for $30 \mathrm{~min}$ followed by centrifugation. Diethyl ether (DTE) was added to the supernatant to the final concentration of $20 \mathrm{mM}$. Then the mixture was heated at $95^{\circ} \mathrm{C}$ for $5 \mathrm{~min}$ and subsequently subjected to $10-16 \%$ SDS-PAGE and immunoblotted on a PVDF membrane (HYBOND LFP PVDF transfer membrane, GE Healthcare, Chalfont St Giles, UK; \#RPN303LFP) using the primary antibodies rabbit anti-DJ-1 (1:2000, Abcam, Cambridge, UK; \#ab18257) and rabbit anti-aSyn (1:1000, described above), as well as HRP conjugated secondary antibodies (1:1000,
\end{abstract} Dako, Glostrup, Denmark).

Immunoprecipitation of endogenous aSyn and DJ-1. C57BL/6 WT mouse (6 months of age) brain homogenate was prepared in RIPA buffer. After retrieving the $10 \%$ input sample, the brain homogenate was incubated with $25 \mu \mathrm{l}$ CNBr-sepharose beads (GE Healthcare, \#17-0430-01) coupled to anti-aSyn (ASY1) or non-immune rabbit $\operatorname{lgG}\left(4 \mathrm{mg} \operatorname{lgG} / \mathrm{ml}\right.$ swelled sepharose) at $4{ }^{\circ} \mathrm{C}$ with rotation for two hours. Then, the beads were isolated, washed, eluted and immunoblotted as described above using the primary antibodies rabbit anti-DJ-1 (1:2000, Abcam \#ab18257) and mouse anti-aSyn (1: 1000, BD \#610787, Franklin Lakes, NJ, USA) and polyclonal swine anti-rabbit $\lg G$ and polyclonal rabbit anti-mouse IgG HRP conjugated secondary antibodies (1:1000, Dako \# P0217 and \#P0260).

Cell culture. HEK293 cells were cultured in DMEM high glucose, supplemented with $10 \%$ fetal calf serum (FCS), 100 units $/ \mathrm{ml}$ penicillin and $100 \mu \mathrm{g} / \mathrm{ml}$ streptomycin; H4 cells were grown in OPTI-MEM, supplemented with $10 \%$ FCS, 100 units $/ \mathrm{ml}$ penicillin and $100 \mu \mathrm{g} / \mathrm{ml}$ streptomycin. $\mathrm{H} 4$ cells stably transfected with two plasmids encoding aSyn tagged with either the N-terminal part or the C-terminal portion of Venus protein ${ }^{5}$ were cultured in OPTI-MEM with $30 \%$ FCS, 100 units $/ \mathrm{ml}$ penicillin, $100 \mu \mathrm{g} / \mathrm{ml}$ streptomycin and $0.1 \%$ G418. All cells were grown at $37^{\circ} \mathrm{C}$ in $95 \%$ air / $5 \% \mathrm{CO}_{2}$ atmosphere. For BiFC experiments, cells were grown on 6-well plates $\left(1.5 \times 10^{5}\right.$ cells/well) pre-coated with $0.01 \%$ Poly-L-lysine solution or on 24 -well plates at a density of $1 \times 10^{4}$ cells/well $24 \mathrm{~h}$ prior to transfection.

Generation of DNA constructs. The aSyn BiFC constructs (VN-Syn, Syn-VC, VN-Syn(A30P), VN-Syn (E46K), VN-Syn(A53T)) have been previously used in our laboratory, ${ }^{5}$ as well as the DJ-1 BiFC constructs (DJ-1-CFPC, DJ-1-GFPN, DJ-1(L10P)-CFPC, DJ-1(M26I)-CFPC, DJ-1(E64D)-CFPC, DJ-1(P158A)-CFPC, DJ-1(L166P)-CFPC)). ${ }^{17}$ The DJ-1(C106A)-CFPC and the DJ-1(K130R)-CFPC constructs were produced as described previously: ${ }^{17}$ the primer sequences are listed in the Supplementary Information; (Supplementary Table S1). To introduce the four different familial point mutations L10P, M26I, P158 1 and L166P into a DJ-1-myc plasmid vector (pGW1-CMV vector backbone), the QuickChange Site-Directed Mutagenesis kit (Stratagene, La Jolla, CA, USA) was employed according to manufacturer protocols using the primers listed in Supplementary Information; (Supplementary Table S2). All constructs were verified by DNA sequencing.
BiFC assays. HEK293 cells were transfected with combinations of DJ-1 and aSyn-BiFC constructs using either Metafectene (Biontex, Munich, Germany) or Effectene (Qiagen, Venlo, Netherlands). Additionally, a transfection control plasmid encoding the red fluorescent mCherry protein or RFP was included in all experiments, allowing the selection of successfully transfected cells. The amount of the mCherry/RFP plasmid comprised one-fifth of the total DNA amount per well, whereas the BiFC constructs were used in equal amounts. The total DNA amount per well was $0.25 \mu \mathrm{g}$ in a 24-well plate format and $0.4 \mu \mathrm{g}$ in a 6-well plate format. To ensure optimal reconstitution of the fluorophores, particularly GFP, the transfected cells were incubated at $30^{\circ} \mathrm{C}$ overnight prior to the image acquisition at the microscope. To explore the effect of DJ-1 on aSyn oligomerization, a BiFCbased aSyn dimerization/oligomerization model developed in our laboratory ${ }^{5}$ was used. $\mathrm{H} 4$ neuroglioma cells stably transfected with Venus-tagged aSyn BiFC constructs were transiently transfected with myc-DJ-1 plasmids using FuGene6 (Promega, Madison, WI, USA), including the same mCherry control plasmid as mentioned above. The total DNA amount per well was $0.5 \mu \mathrm{g}$.

Microscopy. Cells were imaged $24 \mathrm{~h}$ ( $\mathrm{H} 4$ cells) or $48 \mathrm{~h}$ (HEK293 cells) post transfection with an Olympus XI81 system (Olympus Soft Imaging Solutions $\mathrm{GmbH}$, Münster, Germany) or with an Olympus Scan̂R screening station equipped with a $20 \times$ LUCPlanFLN objective $(N A=0.45)$ and a Hamamatsu ORCA-AG CCD camera (Hamamatsu Photomics, Hamamatsu, SZK, Japan). The light source was a MT-20 illumination system (Olympus Soft Imaging Solutions $\mathrm{GmbH}$ ) with a high-stability $150 \mathrm{~W}$ xenon short arc burner. During imaging the cells were kept at $37^{\circ} \mathrm{C}$ and $5 \% \mathrm{CO}_{2}$. GFP/Venus BiFC fluorescence was detected using a $470 /$ $22 \mathrm{~nm}$ excitation filter and a $535 / 50 \mathrm{~nm}$ emission filter. mCherry/RFP expression was imaged using excitation at $556 / 20 \mathrm{~nm}$ and emission between 590 and $650 \mathrm{~nm}$ using the filter set 51019 (Chroma, Bellow Falls, VT, USA). In 24-well format experiments, 25 pictures were taken per well and about $800-1500$ cells were identified to be mCherry positive for each condition in each independent experiment. The ratio between GFP/Venus and mCherry emission intensities was quantified for every cell expressing mCherry after subtraction of the background signal using the Scan̂R analysis software as previously described. ${ }^{17}$ In 6 -well format experiments, 100 pictures were taken per well. All experiments were performed in duplicate (6-well format) or triplicate (24-well format) and repeated three times. To obtain higher magnification representative images for each of the transfection combinations, transfected cells were fixed with 4\% PFA in PBS (PAN) for $10 \mathrm{~min}$ at RT. After a washing step with PBS, cells were incubated for $10 \mathrm{~min}$ at RT in Hoechst 33258 (Molecular Probes, Carlsbad, CA, USA) solution for nuclear staining and then kept in PBS until used for microscopy. Representative images were taken with a Leica epifluorescence microscope (Leica, Wetzlar, Germany) using a $40 \times$ objective.

Immunoblotting of BiFC cell experiments. Directly after image acquisition at the microscope, cells were washed twice with sterile PBS and then lysed on ice for $10 \mathrm{~min}$ in lysis buffer as previously described ${ }^{17}$ or RIPA buffer (50 mM Tris, $150 \mathrm{mM} \mathrm{NaCl}, 1 \%$ Triton X-100, 2 mM EDTA, 0.5\% DOC, 0.1\% SDS, complete protease inhibitor cocktail mix, Roche). Lysates were centrifuged at 13000 r.p.m. for $10 \mathrm{~min}$ at $4^{\circ} \mathrm{C}$. Supernatants were collected and protein concentration was determined by the Bradford method. Samples were stored at $-80^{\circ} \mathrm{C}$ until used. 10 to $30 \mu \mathrm{g}$ of protein per slot were separated on a $12 \%$ SDS polyacrylamide gel and transferred to a polvinylidene difluoride membrane. Membranes were incubated for $1 \mathrm{~h}$ in TBST (50 mM Tris, $150 \mathrm{mM} \mathrm{NaCl}, 0.05 \%$ Tween, $\mathrm{pH} 7.5$ ) containing $5 \%$ milk to saturate all non-specific binding sites. Incubation with primary antibodies was overnight at $4{ }^{\circ} \mathrm{C}$ in $1 \%$ milk + TBST (1: 1500 goat anti-DJ-1, Santa Cruz Biotechnology, Dallas, TX, USA; \#Sc-27006; $1: 3000$ mouse anti- $\alpha$-Synuclein, BD \#610787; 1:5000 mouse anti- $\beta$-actin, Sigma-Aldrich, St. Louis, MO, USA; \#A5441). Blots were developed using horseradish peroxidase (HRP)-conjugated secondary antibodies (anti-goat 1:10000 Jackson Immunoresearch, West Grove, PA, USA; \#705-035-003, anti-mouse 1: 10000 Sigma \#50185-ILM-F) and the Immobilon chemiluminescent HRP substrate (Millipore, Billerica, MA, USA).

Yeast plasmids, strains and culture conditions. Yeast work was performed in the wild-type BY4714 background (MAT, ura3 $\Delta 0$, leu2 $\Delta 0$, his $3 \Delta 1$, met15 $\Delta 0$ ). The yeast expression vector p426GAL was used to express human SNCA fused with GFP under a galactose-inducible promoter (GAL1) as previously described. ${ }^{9}$ Hsp31, Hsp32, Hsp33 or Hsp34 fused to a 6xHis-HA tag, were 
expressed under control of the GAL1 promoter with BG1805 plasmids (Thermo Scientific, Waltham, MA, USA). To express the human DJ-1 gene under the regulation of the constitutive promoter TPI, the yeast vector PYX212 DJ-1 was used. Yeast cultures were transformed using a standard lithium acetate protocol. All strains were grown in synthetic complete medium without uracil and histidine (SC-URA-HIS), $6.7 \mathrm{~g} / \mathrm{l}$ Yeast Nitrogen Base (BD), appropriate amino acid dropout mix (Sunrise Science Products, San Diego, CA, USA), 1\% (w/v) raffinose or $1 \%$ $(\mathrm{w} / \mathrm{v})$ galactose. Yeast cells carrying the galactose-inducible aSyn-GFP construct alone or in combination with empty vector, Hsp31, Hsp32, Hsp33 or Hsp34 were pre-grown in SC-URA-HIS $1 \%$ raffinose for $24 \mathrm{~h}$ and then diluted back in order to reach an optical density $\left(\mathrm{OD}_{600 \mathrm{~nm}}\right)$ of 0.4 . For growth assays on solid medium, the cultures were serially diluted and spotted on SC-URA-HIS containing $1 \%$ galactose or $1 \%$ glucose (control) agar plates, which were then incubated at $30^{\circ} \mathrm{C}$ for at least $48 \mathrm{~h}$. For microscopy studies or protein extraction aSyn expression was induced in SC-URA-HIS $1 \%$ galactose for $7 \mathrm{~h}$.

Yeast protein extraction and immunoblotting. For total protein extraction, yeast cells were lysed in MURB buffer $(50 \mathrm{mM}$ sodium phosphate, $25 \mathrm{mM}$ MES, pH 7.0, 1\% SDS, $3 \mathrm{M}$ urea, 0.5\% 2-mercaptoethanol, $1 \mathrm{mM}$ sodium azide) supplemented with protease and phosphatase inhibitor cocktail (Roche), with glass beads, three cycles of $30 \mathrm{~s}$ in the bead beater and $5 \mathrm{~min}$ on ice. Cell debris was removed by centrifugation at $10000 \mathrm{~g}$ for $1 \mathrm{~min}$ at room temperature and after denaturation at $70^{\circ} \mathrm{C}$, the supernatant was collected. Equal volumes of total protein were analyzed by SDS-PAGE for the detection of aSyn levels. After electrophoresis, samples were transferred to a nitrocellulose membrane using a Trans-Blot Turbo transfer system (Bio-Rad, Hercules, CA, USA). Immunoblotting was performed following standard procedures with antibodies against aSyn (1: 5000, BD \#S63320), PGK (1: 1000, Life Technologies Corporation, Carlsbad, CA, USA; \#459250) and HA (1:1000, Santa Cruz Biotechnology \#sc-7392).

Sucrose gradient analysis of aggregation. Total protein from cells expressing WT or S129A aSyn was obtained and applied on a $5-30 \%$ sucrose gradient as previously described..$^{30}$ Fractions were collected, precipitated for $4 \mathrm{~h}$ at $4^{\circ} \mathrm{C}$ in trichloroacetic acid, washed in acetone three times and suspended in protein sample buffer $(0.5 \mathrm{M}$ Tris- $\mathrm{HCl}$, pH 6.8, glycerol, $10 \%$ (w/v) SDS, $0.1 \%(\mathrm{w} / \mathrm{v})$ bromophenol blue). Proteins were resolved by SDS-PAGE and estimation of the molecular sizes for each fraction were performed as previously described. ${ }^{57}$

Yeast fluorescence microscopy. The percentage of cells with aSyn inclusions was determined by fluorescence microscopy using a Zeiss Axiovert $200 \mathrm{M}$ (Carl Zeiss, Jena, Germany) wide-field fluorescence microscope equipped with a cooled CCD camera (Roper Scientific Coolsnap HQ, Martinsried, Germany) and a $63 \times$ objective to acquire images containing at least 500 cells per strain, which were then counted using ImageJ. Yeast cells were grown as described above. After $7 \mathrm{~h}$ of induction, cells were collected by centrifugation and resuspended in PBS and $0.5 \%$ low melting agarose on a microscope slide.

Statistical analyses. BiFC data were analyzed using the Mann-Whitney non-parametric test (GraphPad Prism 6), whereas yeast data were analyzed by the Tukey HSD (Honest Significant Difference) multiple comparison test $(\alpha=0.05)$. All data are reported as the mean \pm standard error of the mean (S.E.M.). Data were considered significant when $P \leq 0.05$. ${ }^{*}$ indicates $P \leq 0.05$ ${ }^{* *}$ indicates $P \leq 0.01,{ }^{* * *}$ indicates $P \leq 0.001$ and ${ }^{* * * *}$ indicates $P \leq 0.0001$.

\section{Conflict of Interest}

The authors declare no conflict of interest.

Acknowledgements. This work was supported by funding from Parkinson's UK (Research Grant G-0902), Fundação Calouste Gulbenkian and by Fundação para a Ciência e Tecnologia project PTDC/BIA-BCM/117975/2010, and fellowships SFRH/BPD/35767/2007 (ST), SFRH/BD/36065/2007 (LMF), SFRH/BD/79337/ 2011 (SG) and IMM/BTI/91-2012 (RRR). TFO was also supported by an EMBO Installation Grant and by the DFG Center for Nanoscale Microscopy and Molecular Physiology of the Brain (CNMPB).
1. Bonifati V, Rizzu P, Squitieri F, Krieger E, Vanacore N, van Swieten JC et al. DJ-1( PARK7), a novel gene for autosomal recessive, early onset parkinsonism. Neurol Sci 2003; 24: 159-160.

2. Marques O, Outeiro TF. Alpha-synuclein: from secretion to dysfunction and death. Cell Death Dis 2012; 3: e350.

3. Wales $P$, Pinho R, Lazaro DF, Outeiro TF. Limelight on alpha-synuclein: pathological and mechanistic implications in neurodegeneration. J Parkinsons Dis 2013; 3: 415-459.

4. Paleologou KE, El-Agnaf OM. alpha-Synuclein aggregation and modulating factors. Subcell Biochem 2012; 65: 109-164.

5. Outeiro TF, Putcha P, Tetzlaff JE, Spoelgen R, Koker M, Carvalho F et al. Formation of toxic oligomeric alpha-synuclein species in living cells. PloS One 2008; 3: e1867.

6. Winkler S, Hagenah J, Lincoln S, Heckman M, Haugarvoll K, Lohmann-Hedrich K et al. alpha-Synuclein and Parkinson disease susceptibility. Neurology 2007; 69: 1745-1750.

7. Cooper AA, Gitler AD, Cashikar A, Haynes CM, Hill KJ, Bhullar B et al. Alpha-synuclein blocks ER-Golgi traffic and Rab1 rescues neuron loss in Parkinson's models. Science 2006; 313: 324-328.

8. Gitter AD, Bevis BJ, Shorter J, Strathearn KE, Hamamichi S, Su LJ et al. The Parkinson's disease protein alpha-synuclein disrupts cellular Rab homeostasis. Proc Natl Acad Sci USA 2008: 105: 145-150.

9. Outeiro TF, Lindquist S. Yeast cells provide insight into alpha-synuclein biology and pathobiology. Science 2003; 302: 1772-1775.

10. Fasano M, Lopiano L. Alpha-synuclein and Parkinson's disease: a proteomic view. Expert Rev Proteomics 2008; 5: 239-248.

11. Spillantini MG, Schmidt ML, Lee VM, Trojanowski JQ, Jakes R, Goedert M. Alphasynuclein in Lewy bodies. Nature 1997; 388: 839-840.

12. Canet-Aviles RM, Wilson MA, Miller DW, Ahmad R, McLendon C, Bandyopadhyay S et al. The Parkinson's disease protein DJ-1 is neuroprotective due to cysteine-sulfinic acid-driven mitochondrial localization. Proc Natl Acad Sci USA 2004; 101: 9103-9108.

13. Blackinton J, Ahmad R, Miller DW, van der Brug MP, Canet-Aviles RM, Hague SM et al. Effects of DJ-1 mutations and polymorphisms on protein stability and subcellular localization. Brain Research Mol Brain Res 2005; 134: 76-83.

14. Zhang L, Shimoji M, Thomas B, Moore DJ, Yu SW, Marupudi NI et al. Mitochondrial localization of the Parkinson's disease related protein DJ-1: implications for pathogenesis. Hum Mol Genet 2005; 14: 2063-2073.

15. Wilson MA, Collins JL, Hod Y, Ringe D, Petsko GA. The 1.1-A resolution crystal structure of DJ-1, the protein mutated in autosomal recessive early onset Parkinson's disease. Proc Natl Acad Sci USA 2003; 100: 9256-9261.

16. Gorner K, Holtorf E, Waak J, Pham TT, Vogt-Weisenhorn DM, Wurst W et al. Structural determinants of the C-terminal helix-kink-helix motif essential for protein stability and survival promoting activity of DJ-1. J Biol Chem 2007: 282: 13680-13691.

17. Repici M, Straatman KR, Balduccio N, Enguita FJ, Outeiro TF, Giorgini F. Parkinson's disease-associated mutations in DJ-1 modulate its dimerization in living cells. J Mol Med (Berl) 2013; 91: 599-611.

18. Cookson MR. DJ-1, PINK1, and their effects on mitochondrial pathways. Mov Disord 2010; 25: S44-S48.

19. Shendelman S, Jonason A, Martinat C, Leete T, Abeliovich A. DJ-1 is a redox-dependent molecular chaperone that inhibits alpha-synuclein aggregate formation. PLOS Biol 2004; 2. $\mathrm{e} 362$

20. Zhou W, Bercury K, Cummiskey J, Luong N, Lebin J, Freed CR. Phenylbutyrate up-regulates the DJ-1 protein and protects neurons in cell culture and in animal models of Parkinson disease. J Biol Chem 2011; 286: 14941-14951.

21. Sun SY, An CN, Pu XP. DJ-1 protein protects dopaminergic neurons against 6-OHDA/MG-132-induced neurotoxicity in rats. Brain Research Bull 2012; 88: 609-616.

22. Zhou W, Freed CR. DJ-1 up-regulates glutathione synthesis during oxidative stress and inhibits A53T alpha-synuclein toxicity. J Biol Chem 2005; 280: 43150-43158.

23. Mysling S, Betzer C, Jensen PH, Jorgensen TJ. Characterizing the dynamics of alpha-synuclein oligomers using hydrogen/deuterium exchange monitored by mass spectrometry. Biochemistry 2013; 52: 9097-9103.

24. Goncalves SA, Matos JE, Outeiro TF. Zooming into protein oligomerization in neurodegeneration using BiFC. Trends Biochem Sci 2010; 35: 643-651.

25. Hu CD, Kerppola TK. Simultaneous visualization of multiple protein interactions in living cells using multicolor fluorescence complementation analysis. Nat Biotechnol 2003; 21: 539-545.

26. Honbou K, Suzuki NN, Horiuchi M, Niki T, Taira T, Ariga H et al. The crystal structure of DJ-1, a protein related to male fertility and Parkinson's disease. J Biol Chem 2003; 278: 31380-31384

27. Miller-Fleming L, Giorgini F, Outeiro TF. Yeast as a model for studying human neurodegenerative disorders. Biotechnol J 2008; 3: 325-338.

28. Tenreiro S, Munder MC, Alberti S, Outeiro TF. Harnessing the power of yeast to unravel the molecular basis of neurodegeneration. J Neurochem 2013; 127: 438-452.

29. Wilson MA, St Amour CV, Collins JL, Ringe D, Petsko GA. The 1.8-A resolution crystal structure of YDR533Cp from Saccharomyces cerevisiae: a member of the DJ-1/ThiJ/Pfpl superfamily. Proc Natl Acad Sci USA 2004; 101: 1531-1536.

30. Basso E, Antas P, Marijanovic Z, Goncalves S, Tenreiro S, Outeiro TF. PLK2 modulates alpha-synuclein aggregation in yeast and mammalian cells. Mol Neurobiol 2013; 48: 854-862. 
31. Olanow CW, Perl DP, DeMartino GN, McNaught KS. Lewy-body formation is an aggresome-related process: a hypothesis. Lancet Neurol 2004; 3: 496-503.

32. Bonini NM, Giasson BI. Snaring the function of alpha-synuclein. Cell 2005; 123: 359-361.

33. Vekrellis K, Xilouri M, Emmanouilidou E, Rideout HJ, Stefanis L. Pathological roles of alpha-synuclein in neurological disorders. Lancet Neurol 2011; 10: 1015-1025.

34. Cremades N, Cohen SI, Deas E, Abramov AY, Chen AY, Orte A et al. Direct observation of the interconversion of normal and toxic forms of alpha-synuclein. Cell 2012; 149: 1048-1059.

35. Brown DR. Oligomeric alpha-synuclein and its role in neuronal death. IUBMB Life 2010; 62: 334-339.

36. Celej MS, Sarroukh R, Goormaghtigh E, Fidelio GD, Ruysschaert JM, Raussens V. Toxic prefibrillar alpha-synuclein amyloid oligomers adopt a distinctive antiparallel beta-sheet structure. Biochem J 2012; 443: 719-726.

37. Colla E, Jensen PH, Pletnikova O, Troncoso JC, Glabe C, Lee MK. Accumulation of toxic alpha-synuclein oligomer within endoplasmic reticulum occurs in alpha-synucleinopathy in vivo. J Neurosci 2012; 32: 3301-3305.

38. Roostaee A, Beaudoin S, Staskevicius A, Roucou X. Aggregation and neurotoxicity of recombinant alpha-synuclein aggregates initiated by dimerization. Mol Neurodegener 2013; 8: 5.

39. Fan J, Ren H, Jia N, Fei E, Zhou T, Jiang P et al. DJ-1 decreases Bax expression through repressing p53 transcriptional activity. J Biol Chem 2008; 283: 4022-4030.

40. Aleyasin H, Rousseaux MW, Marcogliese PC, Hewitt SJ, Irrcher I, Joselin AP et al. DJ-1 protects the nigrostriatal axis from the neurotoxin MPTP by modulation of the AKT pathway. Proc Natl Acad Sci USA 2010; 107: 3186-3191.

41. Irrcher I, Aleyasin H, Seifert EL, Hewitt SJ, Chhabra S, Phillips M et al. Loss of the Parkinson's disease-linked gene DJ-1 perturbs mitochondrial dynamics. Hum Mol Genet 2010; 19: 3734-3746.

42. Wang X, Petrie TG, Liu Y, Liu J, Fujioka H, Zhu X. Parkinson's disease-associated DJ-1 mutations impair mitochondrial dynamics and cause mitochondrial dysfunction. J Neurochem 2012; 121: 830-839.

43. van Duijn CM, Dekker MC, Bonifati V, Galjaard RJ, Houwing-Duistermaat JJ, Snijders PJ et al. Park7, a novel locus for autosomal recessive early-onset parkinsonism, on chromosome 1p36. Am J Hum Genet 2001; 69: 629-634.

44. Irwin DJ, Lee VM, Trojanowski JQ. Parkinson's disease dementia: convergence of alpha-synuclein, tau and amyloid-beta pathologies. Nat Rev Neurosci 2013; 14: 626-636.

45. Nixon RA. The role of autophagy in neurodegenerative disease. Nat Med 2013; 19 : 983-997.

46. Sajjad MU, Green EW, Miller-Fleming L, Hands S, Herrera F, Campesan S et al. DJ-1 modulates aggregation and pathogenesis in models of Huntington's disease. Hum Mol Genet 2014; 23: 755-766.
47. Zhou W, Zhu M, Wilson MA, Petsko GA, Fink AL. The oxidation state of DJ-1 regulates its chaperone activity toward alpha-synuclein. J Mol Biol 2006; 356: 1036-1048.

48. Girotto S, Cendron L, Bisaglia M, Tessari I, Mammi S, Zanotti G et al. DJ-1 is a copper chaperone acting on SOD1 activation. J Biol Chem 2014; 289: 10887-10899.

49. Yamashita S, Mori A, Kimura E, Mita S, Maeda Y, Hirano T et al. DJ-1 forms complexes with mutant SOD1 and ameliorates its toxicity. J Neurochem 2010; 113 860-870.

50. Jin J, Li GJ, Davis J, Zhu D, Wang Y, Pan C et al. Identification of novel proteins associated with both alpha-synuclein and DJ-1. Mol Cell Proteomics 2007; 6: 845-859.

51. Badiola N, de Oliveira RM, Herrera F, Guardia-Laguarta C, Goncalves SA, Pera M et al. Tau enhances alpha-synuclein aggregation and toxicity in cellular models of synucleinopathy. PLoS One 2011; 6: e26609.

52. Jellinger KA. Interaction between alpha-synuclein and other proteins in neurodegenerative disorders. ScientificWorldJournal 2011; 11: 1893-1907.

53. Nakamura K, Nemani VM, Azarbal F, Skibinski G, Levy JM, Egami K et al. Direct membrane association drives mitochondrial fission by the Parkinson diseaseassociated protein alpha-synuclein. J Biol Chem 2011; 286: 20710-20726.

54. Malgieri G, Eliezer D. Structural effects of Parkinson's disease linked DJ-1 mutations. Protein Sci 2008; 17: 855-868.

55. Ramsey CP, Giasson BI. L10p and P158DEL DJ-1 mutations cause protein instability, aggregation, and dimerization impairments. J Neurosci Res 2010; 88: 3111-3124.

56. Jensen PH, Islam K, Kenney J, Nielsen MS, Power J, Gai WP. Microtubule-associated protein $1 \mathrm{~B}$ is a component of cortical Lewy bodies and binds alpha-synuclein filaments. J Biol Chem 2000; 275: 21500-21507.

57. Tanese N. Small-scale density gradient sedimentation to separate and analyze multiprotein complexes. Methods 1997; 12: 224-234.

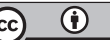

Cell Death and Disease is an open-access journal published by Nature Publishing Group. This work is licensed under a Creative Commons Attribution 3.0 Unported License. The images or other third party material in this article are included in the article's Creative Commons license, unless indicated otherwise in the credit line; if the material is not included under the Creative Commons license, users will need to obtain permission from the license holder to reproduce the material. To view a copy of this license, visit http://creativecommons.org/licenses/by/3.0/

Supplementary Information accompanies this paper on Cell Death and Disease website (http://www.nature.com/cddis) 